\title{
Uniqueness for an Obstacle Problem Arising from Logistic-Type Equations with Fractional Laplacian
}

\author{
Tomasz Klimsiak ${ }^{1,2}$ (1)
}

Received: 1 February 2021 / Accepted: 19 January 2022

(C) The Author(s) 2022

\begin{abstract}
We prove a uniqueness theorem for the obstacle problem for linear equations involving the fractional Laplacian with zero Dirichlet exterior condition. The problem under consideration arises as the limit of some logistic-type equations. Our result extends (and slightly strengthens) the known corresponding results for the classical Laplace operator with zero boundary condition. Our proof, as compared with the known proof for the classical Laplace operator, is entirely new, and is based on the probabilistic potential theory. Its advantage is that it may be applied to a wide class of integro-differential operators.
\end{abstract}

Keywords Dirichlet fractional Laplacian · Obstacle problem · Logistic equation · Intrinsic ultracontractivity

Mathematics Subject Classification (2010) Primary 35R35 · Secondary 35R11

\section{Introduction}

Let $D \subset \mathbb{R}^{d}, d \geq 2$, be a bounded Lipschitz domain, $D_{0} \subset D$ be a bounded Dirichlet regular domain (with respect to the fractional Laplacian). For $\alpha \in(0,2)$, we denote by $\left(\Delta^{\alpha / 2}\right)_{\mid D}$ the Dirichlet fractional Laplacian on $D$ with zero exterior condition (see Section 2 for details). In case $\alpha=2$, by $\left(\Delta^{\alpha / 2}\right)_{\mid D}$ we mean the classical Laplace operator $\Delta_{D}$ on $D$ with zero boundary condition. In the present paper, we prove a uniqueness result for the following obstacle problem:

$$
\left\{\begin{array}{l}
\max \left\{-\left(\Delta^{\alpha / 2}\right)_{\mid D} u-a u, u-\mathbb{I}_{D \backslash \bar{D}_{0}}\right\}=0, \\
u>0, \quad \text { on } \quad D,
\end{array}\right.
$$

Tomasz Klimsiak

tomas@mat.umk.pl

1 Faculty of Mathematics and Computer Science, Nicolaus Copernicus University, ul. Chopina 12/18, 87-100 Toruń, Poland

2 Institute of Mathematics, Polish Academy of Sciences, ul. Śniadeckich 8, 00-656 Warsaw, Poland 
where $a$ is a positive constant, and

$$
\mathbb{I}_{D \backslash \bar{D}_{0}}(x)= \begin{cases}1, & x \in D \backslash \bar{D}_{0}, \\ +\infty, & x \in \bar{D}_{0}\end{cases}
$$

Problem of this type arises in the study of asymptotic behaviour, as $p \rightarrow \infty$, of logistic type equations (see [10,27] for the case $\alpha=2$ and [20] for equations with $\alpha \in(0,2)$ ). Problem (1.1) with $\alpha=2$ also arises as the limit of some predator-prey models (see [8,11]).

From [10] (in case $\alpha=2$ ) and [20] (in case $\alpha \in(0,2)$ ) we know that Eq. 1.1 has a solution if and only if $a \in\left[\lambda_{1}^{D}, \lambda_{1}^{D_{0}}\right)$, where $\lambda_{1}^{D}, \lambda_{1}^{D_{0}}$ stand for the first eigenvalue of $-\left(\Delta^{\alpha / 2}\right)_{\mid D}$ and $-\left(\Delta^{\alpha / 2}\right)_{\mid D_{0}}$, respectively. In the present paper, we concentrate on the uniqueness of solutions to Eq. 1.1. This problem turns out to be difficult, and it has been carefully investigated in [9] in case $\alpha=2$. Suppose that $D_{0}, D$ are smooth and $\bar{D}_{0} \subset D$. The main result of [9] states that Eq. 1.1 has at most one solution if $a \in\left(\lambda_{1}^{D}, \lambda_{1}^{D_{0}}\right)$. This result is proved by using an equivalent free boundary formulation of Eq. 1.1 and tools from the theory of variational inequalities and harmonic functions.

The method used in [9] seems to be suitable only for $\alpha=2$. To deal with nonlocal operators, we propose a new method. It allows us to prove that if $a \in\left(\lambda_{1}^{D}, \lambda_{1}^{D_{0}}\right)$, then for any $\alpha \in(0,2]$ there exists at most one solution to Eq. 1.1. This generalizes the result of [9] to nonlocal operators but also slightly strengthens the known uniqueness result for $\alpha=2$ because we assume that $D_{0} \subset D$ and not that $\bar{D}_{0} \subset D$ as in [9]. Moreover, we consider less regular than in [9] domains $D_{0}, D$ (see comments in [9, Remark (i)]).

In the present paper, we use a definition of a solution to Eq. 1.1, which is equivalent to, but different from that of [9]. Let $\mathcal{E}_{D}$ denote the Dirichlet form associated with the operator $\left(\Delta^{\alpha / 2}\right)_{\mid D}$ (see Section 2). By a solution to Eq. 1.1 we mean a strictly positive function $u \in H_{0}^{\alpha / 2}(D)$ (closure of $C_{c}^{\infty}(D)$ in $H^{\alpha / 2}\left(\mathbb{R}^{d}\right)$ ) having the property that $u \leq \mathbb{I}_{D \backslash \bar{D}_{0}} m$-a.e. and such that for some bounded smooth positive Borel measure $v$ on $D$ (called the reaction measure for $u$ ) we have

$$
\mathcal{E}_{D}(u, v)=a(u, v)-\int_{D} \tilde{v} d v, \quad v \in H_{0}^{\alpha / 2}(D),
$$

where $(\cdot, \cdot)$ stands for the usual inner product in $L^{2}(D ; m)$ and $\tilde{v}$ is a quasi-continuous $m$-version of $v$ ( $m$ stands for the Lebesgue measure on $\mathbb{R}^{d}$ ). Moreover, we require that $u$ satisfies the minimality condition, which says that for every quasi-continuous $\eta$ such that $u \leq \eta \leq \mathbb{I}_{D \backslash \bar{D}_{0}} m$-a.e. we have

$$
\int_{D}(\eta-\tilde{u}) d v=0
$$

where $\tilde{u}$ is a quasi-continuous $m$-version of $u$. In other words, $u$ is a solution of the following equation

$$
-\left(\Delta^{\alpha / 2}\right)_{\mid D} u=a u-v,
$$

where $v$ is a positive measure which acts only when $u$ touches the barrier. Our proof of uniqueness is based on the following two crucial observations. The first one is that if $u$ is a solution to Eq. 1.1, then

$$
u \cdot v=v,
$$

so in fact,

$$
-\left(\Delta^{\alpha / 2}\right)_{\mid D} u=a u-u \cdot v .
$$

Equivalently,

$$
\left(-\left(\Delta^{\alpha / 2}\right)_{\mid D}+v\right) u=a u .
$$


This shows that any solution to Eq. 1.1 is in fact an eigenfunction for the operator $-\left(\Delta^{\alpha / 2}\right)_{\mid D}+v$, i.e. the operator $-\left(\Delta^{\alpha / 2}\right)_{\mid D}$ perturbed by the smooth bounded measure $v$. It is well known that this operator is a self-adjoint positive definite operator on $L^{2}(D ; m)$ generating a Markov $C_{0}$-semigroup of contractions on $L^{2}(D ; m)$. The second crucial observation is that $v$ has a compact support in $D$. This allows us to prove, by using some results of Hansen [16], that the Green function $G_{D}$ for $-\left(\Delta^{\alpha / 2}\right)_{\mid D}$ is comparable with the Green function $G_{D}^{v}$ for $-\left(\Delta^{\alpha / 2}\right)_{\mid D}+v$. These two facts, when combined with the sub and supersolutions method (generalized in the present paper to the case of our obstacle problem), give the uniqueness result.

\section{Potential Theory for Fractional Laplacian on Bounded Domain}

For fixed $x \in \mathbb{R}^{d}$ and $r>0$, we denote $B(x, r)=\left\{y \in \mathbb{R}^{d}:|y-x|<r\right\}$. In the whole paper, we assume that $D$ is an open bounded Lipschitz domain in $\mathbb{R}^{d}$ with Lipschitz character $\left(r_{0}, \lambda\right), r_{0}, \lambda>0$, i.e. open nonempty subset of $\mathbb{R}^{d}$ such that: for every $x \in \partial D$ there exists a function $\Gamma_{x}: \mathbb{R}^{d-1} \rightarrow \mathbb{R}$ such that

$$
\left|\Gamma_{x}(a)-\Gamma_{x}(b)\right| \leq \lambda|a-b|, \quad a, b \in \mathbb{R}^{d-1},
$$

and there exists an orthogonal coordinate system $S_{x}$ such that if $y=\left(y_{1}, y_{2}, \ldots, y_{d}\right)$ in $S_{x}$, then

$$
B\left(x, r_{0}\right) \cap D=B\left(x, r_{0}\right) \cap\left\{y: y_{d}>\Gamma_{x}\left(y_{1}, y_{2}, \ldots, y_{d-1}\right)\right\} .
$$

We denote by $m$ the Lebesgue measure on $\mathbb{R}^{d}$. By $\mathcal{B}\left(\mathbb{R}^{d}\right)$ (resp. $\mathcal{B}(D)$ ), we denote the set of Borel measurable functions on $\mathbb{R}^{d}$ (on $D$ ) with values in $\mathbb{R} \cup\{-\infty\} \cup\{\infty\}$. $\mathcal{B}_{b}(D)$ is the subset of $\mathcal{B}(D)$ consisting of all bounded functions.

\subsection{Dirichlet Fractional Laplacian}

Let $\alpha \in(0,2)$. To define the Dirichlet fractional Laplacian on $D$, we first set

$$
\mathfrak{D}\left(\Delta^{\alpha / 2}\right)=\left\{u \in L^{2}\left(\mathbb{R}^{d} ; m\right): \int_{\mathbb{R}^{d}}|x|^{2 \alpha}|\hat{u}(x)|^{2} m(d x)<\infty\right\},
$$

and for $u \in \mathfrak{D}\left(\Delta^{\alpha / 2}\right)$, we set

$$
\widehat{\Delta^{\frac{\alpha}{2}}} u(x)=|x|^{\alpha} \hat{u}(x), \quad x \in \mathbb{R}^{d},
$$

where $\hat{u}$ stands for the Fourier transform of $u$. Let us consider the form $(\mathcal{E}, \mathfrak{D}(\mathcal{E}))$ defined as

$$
\mathcal{E}(u, v)=\int_{\mathbb{R}^{d}} \hat{u}(x) \hat{v}(x)|x|^{\alpha} m(d x), \quad u, v \in \mathfrak{D}(\mathcal{E}),
$$

where

$$
\mathfrak{D}(\mathcal{E})=H^{\alpha / 2}\left(\mathbb{R}^{d}\right):=\left\{u \in L^{2}\left(\mathbb{R}^{d} ; m\right): \int_{\mathbb{R}^{d}}|x|^{\alpha}|\hat{u}(x)|^{2} m(d x)<\infty\right\} .
$$

By [14, Example 1.4.1], $\left(\mathcal{E}, H^{\alpha / 2}\left(\mathbb{R}^{d}\right)\right)$ is a regular symmetric Dirichlet form on $L^{2}\left(\mathbb{R}^{d} ; m\right)$. When $u \in C_{c}^{\infty}\left(\mathbb{R}^{d}\right)$ the more explicit formula for $-\Delta^{\alpha / 2} u$ is known (see e.g. [14, Example 1.4.1]):

$$
-\Delta^{\alpha / 2} u(x)=c_{d, \alpha} \lim _{r \searrow 0} \int_{\mathbb{R}^{d} \backslash B(x, r)} \frac{u(x)-u(y)}{|x-y|^{d+\alpha}} d y, \quad x \in \mathbb{R}^{d} .
$$


The capacity Cap: $2^{\mathbb{R}^{d}} \rightarrow \mathbb{R}^{+} \cup\{\infty\}$ associated with $\left(\mathcal{E}, H^{\alpha / 2}\left(\mathbb{R}^{d}\right)\right)$ is defined as follows: for an arbitrary open set $U \subset \mathbb{R}^{d}$, we set

$$
\operatorname{Cap}(U)=\inf \left\{\mathcal{E}(u, u), u \in H^{\alpha / 2}\left(\mathbb{R}^{d}\right), u \geq \mathbf{1}_{U} m \text {-a.e. }\right\},
$$

and then, for an arbitrary $B \subset \mathbb{R}^{d}$, we set

$$
\operatorname{Cap}(B)=\inf \left\{\operatorname{Cap}(U): U \supset B, U \subset \mathbb{R}^{d}, U \text { open }\right\} .
$$

We say that some property holds $\mathcal{E}$-quasi everywhere $(\mathcal{E}$-q.e. in abbreviation) if it holds except possibly a set of capacity Cap zero. Such sets shall be called $\mathcal{E}$-exceptional.

Recall that a function $u$ on $\mathbb{R}^{d}$ is called $\mathcal{E}$-quasi-continuous if for every $\varepsilon>0$ there exists a closed set $F_{\varepsilon} \subset \mathbb{R}^{d}$ such that $\operatorname{Cap}\left(\mathbb{R}^{d} \backslash F_{\varepsilon}\right) \leq \varepsilon$ and $u_{\mid F_{\varepsilon}}$ is continuous. By [14, Theorem 2.1.3], each $u \in H^{\alpha / 2}\left(\mathbb{R}^{d}\right)$ admits an $\mathcal{E}$-quasi-continuous $m$-version. In what follows for given function $u \in \mathcal{B}\left(\mathbb{R}^{d}\right)$, we denote by $\tilde{u}$ its $\mathcal{E}$-quasi-continuous $m$-version (whenever it exists).

We denote by $\left(\mathcal{E}_{D}, \mathfrak{D}\left(\mathcal{E}_{D}\right)\right)$ the part of $\left(\mathcal{E}, H^{\alpha / 2}\left(\mathbb{R}^{d}\right)\right)$ on $D$. Recall that $\mathfrak{D}\left(\mathcal{E}_{D}\right)=\left\{u \in H^{\alpha / 2}\left(\mathbb{R}^{d}\right): \tilde{u}=0\right.$, q.e. on $\left.\mathbb{R}^{d} \backslash D\right\}, \quad \mathcal{E}_{D}(u, v)=\mathcal{E}(u, v), \quad u, v \in \mathfrak{D}\left(\mathcal{E}_{D}\right)$. By [14, Theorem 4.4.3], $\left(\mathcal{E}_{D}, \mathfrak{D}\left(\mathcal{E}_{D}\right)\right)$ is a regular symmetric Dirichlet form on $L^{2}(D ; m)$. Therefore, by [14, Sections $1.3,1.4]$, there exists a unique self-adjoint nonpositive definite operator $\left(A_{D}, \mathfrak{D}\left(A_{D}\right)\right)$ on $L^{2}(D ; m)$ such that

$$
\mathfrak{D}\left(A_{D}\right) \subset \mathfrak{D}\left(\mathcal{E}_{D}\right), \quad \mathcal{E}_{D}(u, v)=\left(-A_{D} u, v\right), \quad u \in \mathfrak{D}\left(A_{D}\right), v \in \mathfrak{D}\left(\mathcal{E}_{D}\right) .
$$

The operator $\left(A_{D}, \mathfrak{D}\left(A_{D}\right)\right)$ is called the Dirichlet fractional Laplacian. We put

$$
\left(\Delta^{\alpha / 2}\right)_{\mid D}:=A_{D} .
$$

By [14, Theorem 4.4.3], $C_{c}^{\infty}(D)$ is a dense subspace of $\mathfrak{D}\left(\mathcal{E}_{D}\right)$ in the norm determined by $\mathcal{E}$. Therefore $\mathfrak{D}\left(\mathcal{E}_{D}\right)=H_{0}^{\alpha / 2}(D)\left(\tilde{H}^{\alpha / 2}(D)\right.$ in the notation of [24, page 77]). For $u \in C_{c}^{\infty}(D)$,

$$
\left(\Delta^{\alpha / 2}\right)_{\mid D} u(x)=\Delta^{\alpha / 2} u(x)-\kappa_{D}(x) u(x), \quad x \in D,
$$

where

$$
\kappa_{D}(x)=c_{d, \alpha} \int_{\mathbb{R}^{d} \backslash D}|x-y|^{-d-\alpha} d y, \quad x \in D .
$$

It is worth noting here that $A_{D} \neq-\left(-\Delta_{D}\right)^{\alpha / 2}$, where $\Delta_{D}$ is the Laplace operator with zero Dirichlet boundary condition on $D$. The latter operator is called the fractional Dirichlet Laplacian.

By replacing $\left(\mathcal{E}, H^{\alpha / 2}\left(\mathbb{R}^{d}\right)\right)$ by $\left(\mathcal{E}_{D}, H_{0}^{\alpha / 2}(D)\right)$ and $\mathbb{R}^{d}$ by $D$ in Eqs. 2.1 and 2.2 , we define the capacity $\operatorname{Cap}_{D}$ associated with $\mathcal{E}_{D}$, and then we define the notions of $\mathcal{E}_{D}$-exceptional sets and $\mathcal{E}_{D}$-quasi-continuity. By [14, Theorem 4.4.4], Cap and $\mathrm{Cap}_{D}$ are strongly equivalent on $D$. Therefore, without ambiguity, we may write q.e., exceptional or quasi-continuous instead of $\mathcal{E}$-q.e., $\mathcal{E}_{D}$-q.e. $\mathcal{E}$-exceptional, $\mathcal{E}_{D}$-exceptional or $\mathcal{E}$-quasi-continuous, $\mathcal{E}_{D}$-quasi-continuous.

\subsection{Green Functions and Transition Functions}

We denote by $\left(J_{\beta}\right)_{\beta>0},\left(T_{t}\right)_{t \geq 0}$ the resolvent and the $C_{0}$-semigroup of contractions generated by $A:=\Delta^{\alpha / 2}$, respectively, and by $\left(J_{\beta}^{D}\right)_{\beta>0},\left(T_{t}^{D}\right)_{t \geq 0}$ the resolvent and the $C_{0}$-semigroup of contractions generated by $\left(\Delta^{\alpha / 2}\right)_{\mid D}$, respectively.

Let $\partial$ be a one-point compactification of $D$. Let $\mathcal{D}$ denote the set of all functions $\omega$ : $[0, \infty) \rightarrow \mathbb{R}^{d} \cup\{\partial\}$, that are right continuous and possess the left limits for all $t \geq 0$ 
(cádlágs), and have the property that if $\omega(t)=\partial$, then $\omega(s)=\partial, s \geq t$. We equip $\mathcal{D}$ with the Skorokhod topology, see e.g. Section 12 of [1]. Define the canonical process: $X: \mathcal{D} \rightarrow \mathcal{D}, X_{t}(\omega):=\omega(t), \omega \in \mathcal{D}$, shift operator: $\theta_{s}: \mathcal{D} \rightarrow \mathcal{D}, \theta_{s}(\omega)(t)=\omega(t+s)$, and life time: $\zeta: \mathcal{D} \rightarrow[0, \infty], \zeta(\omega):=\inf \left\{t>0: X_{t}(\omega)=\partial\right\}$. It is well known that there exists a rotation invariant $\alpha$-stable Lévy process $\mathbb{X}=\left(X,\left(P_{x}\right)_{x \in \mathbb{R}^{d}},\left(\mathcal{F}_{t}\right)_{t \geq 0}\right)$ on $\mathbb{R}^{d}$ such that for every positive $f \in \mathcal{B}\left(\mathbb{R}^{d}\right)$,

$$
T_{t} f(x)=\mathbb{E}_{x} f\left(X_{t}\right):=\int_{\mathcal{D}} f\left(X_{t}(\omega)\right) d P_{x}(\omega), \quad x \in \mathbb{R}^{d}, t \geq 0, \quad m \text {-a.e. }
$$

We let for $\beta>0$ and $f \in \mathcal{B}^{+}\left(\mathbb{R}^{d}\right)$

$$
R_{\beta} f(x)=\mathbb{E}_{x} \int_{0}^{\infty} e^{-\beta t} f\left(X_{t}\right) d t, \quad x \in \mathbb{R}^{d} .
$$

In the whole paper we adopt the convention that any function $f$ defined on a subset of $\mathbb{R}^{d}$ equals zero at $\partial$. By [14, Theorem 4.4.2], there exists a Hunt process $\mathbb{X}^{D}=$ $\left(X,\left(P_{x}^{D}\right)_{x \in D \cup\{\partial\}},\left(\mathcal{F}_{t}\right)_{t \geq 0}\right)$ on $D \cup\{\partial\}$ such that

$$
T_{t}^{D} f(x)=\mathbb{E}_{x}^{D} f\left(X_{t}\right):=\int_{\mathcal{D}} f\left(X_{t}(\omega)\right) d P_{x}^{D}(\omega), \quad x \in D, t \geq 0, \quad m \text {-a.e. }
$$

Here $\left(\mathcal{F}_{t}\right)_{t \geq 0}$ is a filtration (non-decreasing sequence of $\sigma$-fields of subsets of $\Omega$ ). By [14, Theorem 4.4.1, Theorem 4.4.2], for all $t \geq 0$ and positive $f \in \mathcal{B}(D)$,

$$
P_{t}^{D} f(x):=\mathbb{E}_{x}^{D} f\left(X_{t}\right)=\mathbb{E}_{x}\left[f\left(X_{t}\right) \mathbf{1}_{\left\{t<\tau_{D}\right\}}\right], \quad x \in D,
$$

where

$$
\tau_{D}=\inf \left\{t>0: X_{t} \in \mathbb{R}^{d} \backslash D\right\}
$$

Hence

$$
R_{\beta}^{D} f(x):=\mathbb{E}_{x}^{D} \int_{0}^{\infty} e^{-\beta t} f\left(X_{t}\right) d t=\mathbb{E}_{x} \int_{0}^{\tau_{D}} e^{-\beta t} f\left(X_{t}\right) d t, \quad x \in D .
$$

It is well known (see [14, Exercise 4.2.1, Lemma 4.2.4]) that there is $G_{\beta} \in \mathcal{B}\left(\mathbb{R}^{d}\right) \times \mathcal{B}\left(\mathbb{R}^{d}\right.$ ) (called the $\beta$-Green function) such that for every $f \in \mathcal{B}^{+}\left(\mathbb{R}^{d}\right)$,

$$
R_{\beta} f(x)=\int_{\mathbb{R}^{d}} G_{\beta}(x, y) f(y) d y, \quad x \in \mathbb{R}^{d} .
$$

Similarly, there is $G_{D, \beta} \in \mathcal{B}(D) \times \mathcal{B}(D)$ (called the $\beta$-Green function for $D$ ) such that for every $f \in \mathcal{B}^{+}(D)$,

$$
R_{\beta}^{D} f(x)=\int_{D} G_{D, \beta}(x, y) f(y) d y, \quad x \in D .
$$

Given a positive Borel measure $\mu$ on $D$, we set

$$
R_{\beta}^{D} \mu(x)=\int_{D} G_{D, \beta}(x, y) \mu(d y), \quad x \in D .
$$

Note that $R_{\beta}^{D} f=J_{\beta}^{D} f m$-a.e. for every $f \in L^{2}(D ; m)$. Moreover, by [14, Theorem 4.2.3], $J_{\beta}^{D} f \in H_{0}^{\alpha / 2}(D)$ and $\widetilde{J_{\beta}^{D} f}=R_{\beta}^{D} f$ q.e. for $f \in L^{2}(D ; m)$. By [14, Exercise 4.2.1], there exists a transition function $p_{D}: \mathbb{R}^{+} \times D \times D \rightarrow \mathbb{R}^{+}$such that for every $f \in \mathcal{B}^{+}(D)$,

$$
P_{t}^{D} f(x)=\int_{D} p_{D}(t, x, y) f(y) d y \quad x \in D, t>0 .
$$


It is well known (see e.g. [15]) that $p_{D}(t, x, y)$ is finite and strictly positive for $x, y \in$ $D, t>0$. Given a positive Borel measure $\mu$ on $D$, we set

$$
P_{t}^{D} \mu(x)=\int_{D} p_{D}(t, x, y) \mu(d y), \quad x \in D, t>0 .
$$

By [14, Theorem 4.2.3], $T_{t}^{D} f \in H_{0}^{\alpha / 2}(D)$ and $\widetilde{T_{t}^{D} f}=P_{t}^{D} f$ q.e. for all $f \in L^{2}(D ; m)$ and $t>0$. Moreover,

$$
G_{D, \beta}(x, y)=\int_{0}^{\infty} e^{-\beta t} p_{D}(t, x, y) d t, \quad x, y \in D .
$$

It follows that we can set

$$
G_{D}(x, y):=\sup _{\beta>0} G_{D, \beta}(x, y)=\lim _{\beta \searrow 0} G_{D, \beta}(x, y), \quad x, y \in D .
$$

It is well known that $\left(\Delta^{\alpha / 2}\right)_{\mid D}: \mathfrak{D}\left(\left(\Delta^{\alpha / 2}\right)_{\mid D}\right) \rightarrow L^{2}(D ; m)$ is a bijection. From the above definition of $G_{D}$ it follows that for every $f \in L^{2}(D ; m)$,

$$
J^{D} f:=\left(-\left(\Delta^{\alpha / 2}\right)_{\mid D}\right)^{-1} f=\int_{D} G_{D}(\cdot, y) f(y) d y \quad m \text {-a.e. on } D .
$$

By [14, Lemma 2.2.11], $J^{D} f \in H_{0}^{\alpha / 2}(D)$ and $\widetilde{J^{D} f}=R^{D} f$ q.e. for every $f \in L^{2}(D ; m)$. For a positive Borel measure $\mu$ on $D$, we set

$$
R^{D} \mu(x)=\int_{D} G_{D}(x, y) \mu(d y), \quad x \in D .
$$

Recall that a positive Borel measurable function $u$ on $D$ is called excessive if

$$
\sup _{t>0} P_{t}^{D} f(x)=f(x), \quad x \in D .
$$

By [14, Lemma 4.2.4], for every positive Borel measure $\mu, R^{D} \mu$ is an excessive function.

\subsection{Smooth Measures}

An increasing sequence $\left\{F_{n}\right\}$ of closed subsets of $\mathbb{R}^{d}$ (resp. $D$ ) is called a generalized $\mathcal{E}$-nest (resp. generalized $\mathcal{E}_{D}$-nest) if for every compact $K \subset \mathbb{R}^{d}$ (resp. $K \subset D$ ), $\operatorname{Cap}\left(K \backslash F_{n}\right) \rightarrow 0$ $\left(\right.$ resp. $\operatorname{Cap}_{D}\left(K \backslash F_{n}\right) \rightarrow 0$ ) as $n \rightarrow \infty$. A Borel signed measure $\mu$ on $\mathbb{R}^{d}$ (resp. $D$ ) is called $\mathcal{E}$-smooth (resp. $\mathcal{E}_{D}$-smooth) if it charges no set of capacity Cap (resp. Cap ${ }_{D}$ ) zero and there exists a generalized $\mathcal{E}$-nest (resp. $\mathcal{E}_{D}$-nest) such that $|\mu|\left(F_{n}\right)<\infty, n \geq 1$, where $|\mu|$ denotes the variation of $\mu$. Note that if a Borel measure $\mu$ on $D$ is bounded, then it is $\mathcal{E}$-smooth if and only if it is $\mathcal{E}_{D}$-smooth. This follows from the fact that Cap and $\operatorname{Cap}_{D}$ are equivalent.

We denote by $\mathcal{M}_{0}\left(\right.$ resp. $\left.\mathcal{M}_{0}(D)\right)$ the set of all $\mathcal{E}$-smooth (resp. $\mathcal{E}_{D^{-}}$-smooth) measures on $\mathbb{R}^{d}$ (resp. $D$ ). We also set

$\mathbb{M}_{0}(D)=\left\{\mu \in \mathcal{M}_{0}(D): R^{D}|\mu|<\infty\right.$ q.e. $\}, \quad M_{0, b}(D)=\left\{\mu \in \mathcal{M}_{0}(D):|\mu|(D)<\infty\right\}$.

By [21, Proposition 5.13],

$$
\mathcal{M}_{0, b}(D) \subset \mathbb{M}_{0}(D) .
$$

Also note that if $\mu$ is a Borel measure such that $R^{D}|\mu|<\infty$ on $D$, then $\mu \in \mathcal{M}_{0}(D)$ (see [14, Exercise 4.2.2]). We say that an $\mathcal{E}_{D}$-smooth measure $\mu$ belongs to the class $S_{0}(D)$ 
(called the class of measures of finite energy integral) if there exists $c>0$ such that

$$
\int_{D}|\tilde{u}| d|\mu| \leq c \sqrt{\mathcal{E}_{D}(u, u)}, \quad u \in H_{0}^{\alpha / 2}(D) .
$$

By [14, Theorem 2.2.2], for any positive $\mu \in S_{0}(D)$ and $\eta \in H_{0}^{\alpha / 2}(D)$,

$$
\mathcal{E}_{D}\left(R^{D} \mu, \eta\right)=\int_{D} \tilde{\eta} d \mu
$$

\subsection{Probabilistic Potential Theory}

Recall that a bounded subset $D$ of $\mathbb{R}^{d}$ is called Dirichlet regular (with respect to the fractional Laplacian) if for every $x \in \mathbb{R}^{d} \backslash D$,

$$
P_{x}\left(\tau_{D}>0\right)=0 .
$$

By [2, Example VII.3.4.3], each bounded Lipschitz domain is Dirichlet regular. From this and [5] it follows that $\left(P_{t}^{D}\right)_{t \geq 0}$ is doubly Feller. This means that it is strongly Feller, i.e. $P_{t}^{D} f \in C_{b}(D)$ for every $f \in \mathcal{B}_{b}(D)$, and it is Fellerian, i.e. $P_{t}^{D} f \in C_{0}(D)$ for every $f \in C_{0}(D)$.

By [14, Theorem 5.1.4], there is a one-to-one correspondence between positive $\mathcal{E}_{D^{-}}$ smooth measures and positive continuous additive functionals (PCAFs) of $\mathbb{X}^{D}$ (see [14, Section 5.1]). By $A^{\mu}$ we denote the unique PCAF of $\mathbb{X}^{D}$ associated with $\mu \in \mathcal{M}_{0}(D)$. By [14, Theorem 5.1.3] and Eq. 2.6,

$$
\mathbb{E}_{x}^{D} \int_{0}^{\infty} f\left(X_{r}\right) d A_{r}^{\mu}=\mathbb{E}_{x} \int_{0}^{\tau_{D}} f\left(X_{r}\right) d A_{r}^{\mu}=\int_{D} G_{D}(x, y) f(y) \mu(d y)=R^{D}(f \cdot \mu)(x)
$$

for q.e. $x \in D$. For a signed measure $\mu \in \mathcal{M}_{0}(D)$ having a decomopsition $\mu=\mu^{+}-\mu^{-}$ into a positive and negative part, we set $A^{\mu}=A^{\mu^{+}}-A^{\mu^{-}}$. Note also that if $\mu \in \mathcal{M}_{0, b}^{+}(D)$ and $\sup _{x \in D} R^{D} \mu<\infty$, then there exists a strict PCAF $A^{\mu}$ of $\mathbb{X}^{D}$ such that Eq. 2.9 holds for every $x \in D$ (see [14, Theorem 5.1.6]).

Recall that a càdlàg process $M$ adapted to $\left(\mathcal{F}_{t}\right)_{t \geq 0}$ is called a martingale additive functional (MAF) of $\mathbb{X}^{D}$ iff $M_{0}=0, M$ is an additive functional, $\mathbb{E}_{x}^{D}\left|M_{t}\right|<\infty$ q.e. $t \geq 0$, and $\mathbb{E}_{x}^{D} M_{t}=0$ q.e. $t \geq 0$. A MAF $M$ of $\mathbb{X}^{D}$ is called uniformly integrable iff for q.e. $x \in D$, and every stopping time $\alpha \leq \tau_{D}$,

$$
\mathbb{E}_{x}^{D}\left|M_{\alpha}\right|<\infty, \quad \mathbb{E}_{x}^{D}\left(M_{\tau_{D}} \mid \mathcal{F}_{\alpha}\right)=M_{\alpha}, \quad P_{x}^{D} \text {-a.s. }
$$

The following very useful result will be frequently used in the paper.

Lemma 2.1 Assume that $u \in \mathcal{B}(D)$ and $\mu \in \mathbb{M}_{0}(D)$. Then

$$
u(x)=R^{D} \mu(x)
$$

for q.e. $x \in D$ if and only if there exists a uniformly integrable MAF $M$ of $\mathbb{X}^{D}$ such that for q.e. $x \in D$,

$$
u\left(X_{t}\right)=\int_{t}^{\tau_{D}} d A_{r}^{\mu}-\int_{t}^{\tau_{D}} d M_{r}, \quad t \in\left[0, \tau_{D}\right], \quad P_{x} \text {-a.s. }
$$

Moreover, if $\sup _{x \in D} R^{D}|\mu|(x)<\infty$, then "for q.e. $x \in D$ " may be replaced by "for every $x \in D$ ” with $A^{\mu}$ being a strict PCAF of $\mathbb{X}^{D}$. 
Proof First suppose that Eq. 2.11 is satisfied. Taking the expectation with respect to $P_{x}$ of both sides of Eq. 2.11 with $t=0$, and then using (2.9) with $f=1$ we get (2.10). Now suppose that Eq. 2.10 holds. Let $N$ be an exceptional set such that Eq. 2.10 holds for $x \in D \backslash N$. By [14, Theorem 4.1.1], we may assume that $P_{x}^{D}\left(X_{t} \notin N, t \geq 0\right)=1$ for every $x \in D \backslash N$. Hence, by Eq. 2.10, additivity of $A^{\mu}$, and strong Markov property, for every $x \in D \backslash N$ and every stopping time $\sigma \leq \tau_{D}$, we have

$$
u\left(X_{\sigma}\right)=\mathbb{E}_{X_{\sigma}}^{D} \int_{0}^{\infty} d A_{r}^{\mu}=\mathbb{E}_{x}^{D}\left(\int_{\sigma}^{\infty} d A_{r}^{\mu} \mid \mathcal{F}_{\sigma}\right)=\mathbb{E}_{x}^{D}\left(\int_{0}^{\infty} d A_{r}^{\mu} \mid \mathcal{F}_{\sigma}\right)-A_{\sigma}^{\mu} \quad P_{x}^{D} \text {-a.s. }
$$

Set

$$
M_{t}=u\left(X_{t}\right)-u\left(X_{0}\right)+A_{t}^{\mu}, \quad t \geq 0 .
$$

By quasi-continuity of $u$ and [14, Theorem 4.6.1], $M$ is a càdlàg process. Clearly, $M$ is an additive functional and $M_{0}=0$. By Eq. 2.12, $M$ is a uniformly integrable martingale on $\left[0, \tau_{D}\right]$ under measure $P_{x}^{D}$. Equivalently, by Eq. $2.5, M$ is a uniformly integrable martingale on $\left[0, \tau_{D}\right]$ under measure $P_{x}$ for $x \in D \backslash N$. If $\sup _{x \in D} R^{D}|\mu|<\infty$, then the above argument holds true with $N=\emptyset$ with one exception that instead of [14, Theorem 4.6.1] one need to apply [3, Theorem III.5.7].

\subsection{Feynman-Kac Formula}

For a positive measure $\mu \in \mathcal{M}_{0, b}(D)$ such that $\sup _{x \in D} R^{D} \mu(x)<\infty$, we define the perturbation of the form $\left(\mathcal{E}_{D}, H_{0}^{\alpha / 2}(D)\right)$ by $\mu$ as follows:

$$
\mathfrak{D}\left(\mathcal{E}_{D}^{\mu}\right)=\left\{u \in H_{0}^{\alpha / 2}(D): \int_{D} \tilde{u}^{2} d \mu<\infty\right\}, \quad \mathcal{E}_{D}^{\mu}(u, v)=\mathcal{E}_{D}(u, v)+\int_{D} \tilde{u} \tilde{v} d \mu .
$$

By [14, Theorem 6.1.2], $\left(\mathcal{E}_{D}^{\mu}, \mathfrak{D}\left(\mathcal{E}_{D}^{\mu}\right)\right)$ is a regular symmetric Dirichlet form on $L^{2}(D ; m)$. Let $\left(T_{t}^{D, \mu}\right)_{t>0}$ be the semigroup generated by $\mathcal{E}_{D}^{\mu}$ and $A_{D}^{\mu}$ be its generator. We let

$$
-\left(\Delta^{\alpha / 2}\right)_{\mid D}+\mu:=-A_{D}^{\mu} \text {. }
$$

By [14] there exists a Hunt process $\mathbb{X}^{D, \mu}=\left(X,\left(P_{x}^{D, \mu}\right)_{x \in D \cup\{\partial\}},\left(\mathcal{F}_{t}\right)_{t \geq 0}\right)$ on $D \cup\{\partial\}$ such that

$$
T_{t}^{D, \mu} f(x)=\mathbb{E}_{x}^{D, \mu} f\left(X_{t}\right):=\int_{\mathcal{D}} f\left(X_{t}(\omega)\right) d P_{x}^{D, \mu}(\omega), \quad x \in D, t \geq 0, \quad m \text {-a.e. }
$$

We denote by $G_{D}^{\mu}$ the Green function for $-\left(\Delta^{\alpha / 2}\right)_{\mid D}+v$ (see [14, Exercise 6.1.1]). By [14, Theorem 6.1.1], we have the Feynman-Kac representation formula

$$
\mathbb{E}_{x}^{D, \mu} f\left(X_{t}\right)=\mathbb{E}_{x}\left[e^{-A_{t}^{\mu}} f\left(X_{t}\right) \mathbf{1}_{\left\{t<\tau_{D}\right\}}\right], \quad x \in D, t \geq 0 .
$$

\section{Integral Supersolutions of the Obstacle Problem}

Let $h: D \rightarrow \mathbb{R} \cup\{\infty\}$ be a measurable strictly positive function and $f: \mathbb{R} \rightarrow \mathbb{R}$ be a continuous function. Consider the following obstacle problem:

$$
\left\{\begin{array}{l}
\max \left\{-\left(\Delta^{\alpha / 2}\right)_{\mid D} u-f(u), u-h\right\}=0, \\
u>0 \text { on } D .
\end{array}\right.
$$

In this section, we give a definition of an integral solution to Eq. 3.1. We also recall widely used in the literature definition of weak solutions via variational inequalities. We show that 
both definitions are equivalent under regularity assumptions on $u$. However, we prefer to work on integral solutions. The advantage of this notion is that its formulation does not require any regularity from solutions (merely integrability). Moreover, by using integral solutions, we may apply probabilistic potential theory, which is very useful in many reasonings. We then show that minimum of two integral supersolutions of Eq. 3.1 is again an integral supersolution. This property shall be one of the crucial ingredients in the proof of uniqueness of Eq. 1.1.

Definition 3.1 We say that a quasi-continuous function $u \in L^{1}(D ; m)$ is an integral solution to Eq. 3.1 if there exists a positive $v \in \mathbb{M}_{0}(D)$ (we call it the reaction measure for $u$ ) such that

(a) $0<u \leq h m$-a.e. and $R^{D}|f(u)|<\infty$ q.e.,

(b) For q.e. $x \in D$,

$$
u(x)=R^{D} f(u)(x)-R^{D} v(x),
$$

(c) For any quasi-continuous function $\eta$ on $D$ such that $u \leq \eta \leq h m$-a.e.,

$$
\int_{D}(\eta-u) d v=0
$$

Remark 3.2 (i) Take any quasi-continuous function $\hat{h}$ such that $u \leq \hat{h} \leq h m$-a.e. Observe that $u$ is an integral solution to Eq. 3.1 if and only if $u$ is an integral solution to Eq. 3.1 with $h$ replaced by $\hat{h}$.

(ii) If $h \in H_{0}^{\alpha / 2}(D)$ is quasi-continuous, then $u$ is an integral solution to Eq. 3.1 if and only if (a), (b) are satisfied and $\int_{D}(h-u) d v=0$.

(iii) Recall that by [14, Lemma 2.1.4], if $u \leq \eta, m$-a.e. and $u, \eta$ are quasi-continuous, then $u \leq \eta$ q.e.

(iv) Note that by [14, Theorem 4.6.1,Theorem A.2.7], functions $R^{D} f^{+}(u), R^{D} f^{-}(u)$, $R^{D} v$ are quasi-continuous as all the mentioned functions are excessive and finite q.e. So, if (b) holds $m$-a.e., then by (iii), (b) holds q.e. Clearly, $R^{D} f^{+}(u), R^{D} f^{-}(u)$ does not depend on the version of $u$. Therefore, if we assume in the Definition 3.1 that $u$ is merely a measurable function such that (a), (c) hold, and (b) holds $m$-a.e., then there exists a version $\tilde{u}$ of $u$ such that $\tilde{u}$ is quasi-continuous and (b) holds q.e. with $u$ replaced by $\tilde{u}$ : this version is given by $\tilde{u}(x):=R^{D} f(u)(x)-R^{D} v(x)$ if $R^{D}|f|(u)(x)+R^{D} v(x)<\infty$ and zero otherwise. Clearly, $\tilde{u}=R^{D} f(\tilde{u})-R^{D} v$ q.e.

Recall that $(\cdot, \cdot)$ stands for the usual inner product in $L^{2}(D ; m)$.

Definition 3.3 We say that a quasi-continuous function $u \in H_{0}^{\alpha / 2}(D)$ is a weak solution to Eq. 3.1 if

(a) $0<u \leq h m$-a.e. and $f(u) \in L^{2}(D ; m)$,

(b) For every $\eta \in H_{0}^{\alpha / 2}(D)$ such that $\eta \leq h m$-a.e.,

$$
\mathcal{E}_{D}(u, \eta-u) \geq(f(u), \eta-u) .
$$

Proposition 3.4 Let $u \in H_{0}^{\alpha / 2}(D)$ and $f(u) \in L^{2}(D ; m)$. Then $u$ is an integral solution to Eq. 3.1 iff $u$ is a weak solution to Eq. 3.1. 
Proof Let $u$ be an integral solution to Eq. 3.1. Since $f(u) \in L^{2}(D ; m), R^{D} f(u) \in$ $\mathfrak{D}\left(\left(\Delta^{\alpha / 2}\right)_{\mid D}\right) \subset H_{0}^{\alpha / 2}(D)$. Thus, $v \in S_{0}(D)$. Therefore, by Eq. 2.8 and (c) of Definition 3.1 , for every $\eta \in H_{0}^{\alpha / 2}(D)$ such that $\eta \leq h m$-a.e.,

$\mathcal{E}_{D}(u, \eta-u)=\mathcal{E}_{D}\left(R^{D} f(u)-R^{D} v, \eta-u\right)=(f(u), \eta-u)-\int_{D}(\tilde{\eta}-\tilde{u}) d v \geq(f(u), \eta-u)$.

Now, let $u$ be a weak solution to Eq. 3.1. By [23, Theorem 5.2, Chapter 3] $u_{n} \rightarrow u$ weakly in $H_{0}^{\alpha / 2}(D)$, where $u_{n} \in H_{0}^{\alpha / 2}(D)$ is a unique solution to the following variational equality

$$
\mathcal{E}_{D}\left(u_{n}, \eta\right)=(f(u), \eta)-\left(n\left(u_{n}-h\right)^{+}, \eta\right), \quad \eta \in H_{0}^{\alpha / 2}(D) .
$$

Clearly, $u_{n}=R^{D} f(u)-R^{D} v_{n}, m$-a.e., where $v_{n}=n\left(u_{n}-h\right)^{+} \cdot m$. By [19, Theorem 3.8], $u_{n} \searrow w$, where $w$ is an integral solution to

$$
\max \left\{-\left(\Delta^{\alpha / 2}\right)_{\mid D} w-f(u), w-h\right\}=0 .
$$

Since $u=w, m$-a.e., we get the desired result (cf. Remark 3.2(iv)).

Definition 3.5 We say that a quasi-continuous function $u \in L^{1}(D ; m)$ is an integral supersolution (resp. integral subsolution) to Eq. 3.1 if there exists a positive measure $v \in \mathbb{M}_{0}(D)$ and a positive (resp. negative) measure $\mu \in \mathbb{M}_{0}(D)$ such that conditions (a) and (c) of Definition 3.1 are satisfied, and moreover, for q.e. $x \in \mathbb{R}^{d}$,

$$
u(x)=R^{D} f(u)(x)+R^{D} \mu(x)-R^{D} v(x) .
$$

Proposition 3.6 If $u$ is an integral supersolution and an integral subsolution to Eq. 3.1, then $u$ is an integral solution to Eq. 3.1.

Proof By assumptions and Definition 3.5, there exist positive measures $v_{1}, v_{2}, \mu_{1}, \mu_{2} \in$ $\mathbb{M}_{0}(D)$ such that

$$
\begin{aligned}
& u(x)=R^{D} f(u)(x)+R^{D} \mu_{1}(x)-R^{D} v_{1}(x), \\
& u(x)=R^{D} f(u)(x)-R^{D} \mu_{2}(x)-R^{D} v_{2}(x),
\end{aligned}
$$

and $\int_{D}(\eta-u) d v_{1}=\int_{D}(\eta-u) d \nu_{2}=0$ for every quasi-continuous $\eta$ on $D$ such that $u \leq \eta \leq h m$-a.e. Thus,

$$
R^{D} v_{1}(x)-R^{D} v_{2}(x)=R^{D} \mu_{1}(x)+R^{D} \mu_{2}(x)
$$

for q.e. $x \in D$. From this, we conclude that $v_{1}-v_{2}=\mu_{1}+\mu_{2}$. Therefore, there exist positive $\alpha, \beta, \gamma \in \mathcal{B}(D)$ such that $\alpha+\beta=1, \gamma \leq 1$ and

$$
v_{2}=\gamma \cdot v_{1}, \quad \mu_{1}=\alpha(1-\gamma) \cdot v_{1}, \quad \mu_{2}=\beta(1-\gamma) \cdot v_{1} .
$$

Consequently, for q.e $x \in D$,

$$
u(x)=R^{D} f(u)(x)-R^{D}\left((\beta+\alpha \gamma) \cdot v_{1}\right)(x) .
$$

Since $\int_{D}(\eta-u)(\beta+\alpha \gamma) d \nu_{1}=0$ for every quasi-continuous $\eta$ on $D$ such that $u \leq \eta \leq h$ $m$-a.e., we see that $u$ is an integral solution to Eq. 3.1.

Proposition 3.7 Let $u_{1}, u_{2}$ be integral supersolutions to Eq. 3.1. Then $u_{1} \wedge u_{2}$ is a an integral supersolution to Eq. 3.1. 
Proof By the definition of a an integral supersolution to Eq. 3.1 and Lemma 2.1, for q.e. $x \in D$, we have

$u_{i}\left(X_{t}\right)=\int_{t}^{\tau_{D}} d A_{r}^{\mu_{i}}+\int_{t}^{\tau_{D}} f\left(u\left(X_{r}\right)\right) d r-\int_{t}^{\tau_{D}} d A_{r}^{v_{i}}-\int_{t}^{\tau_{D}} d M_{r}^{i}, \quad t \in\left[0, \tau_{D}\right], \quad P_{x}$-a.s.

$i=1,2$, for some positive $v_{1}, v_{2}, \mu_{1}, \mu_{2} \in \mathbb{M}_{0}(D)$ and some uniformly integrable MAFs $M^{1}, M^{2}$ of $\mathbb{X}^{D}$. By the Tanaka-Meyer formula (see, e.g., [25, IV.Theorem 70]) applied to $u_{1}(X)-\left(u_{1}(X)-u_{2}(X)\right)^{+}=u_{1}(X) \wedge u_{2}(X)$, there exists an increasing càdlàg process $C$ with $C_{0}=0$ such that for q.e. $x \in D$,

$$
\begin{aligned}
\left(u_{1} \wedge u_{2}\right)\left(X_{t}\right)= & \left(u_{1} \wedge u_{2}\right)(x)-C_{t} \\
& -\int_{0}^{t} \mathbf{1}_{\left\{u_{1}\left(X_{r}\right)>u_{2}\left(X_{r}\right)\right\}} f\left(u_{2}\left(X_{r}\right)\right) d r-\int_{0}^{t} \mathbf{1}_{\left\{u_{1}\left(X_{r}\right)>u_{2}\left(X_{r}\right)\right\}}\left(d A_{r}^{\mu_{2}}-d A_{r}^{v_{2}}\right) \\
& -\int_{0}^{t} \mathbf{1}_{\left\{u_{2}\left(X_{r}\right) \geq u_{1}\left(X_{r}\right)\right\}} f\left(u_{1}\left(X_{r}\right)\right) d r-\int_{0}^{t} \mathbf{1}_{\left\{u_{2}\left(X_{r}\right) \geq u_{2}\left(X_{r}\right)\right\}}\left(d A_{r}^{\mu_{1}}-d A_{r}^{v_{1}}\right) \\
& +\int_{0}^{t} \mathbf{1}_{\left\{u_{1}\left(X_{r-}\right)>u_{2}\left(X_{r-}\right)\right\}} d M_{r}^{2}+\int_{0}^{t} \mathbf{1}_{\left\{u_{2}\left(X_{r-}\right) \geq u_{2}\left(X_{r-}\right)\right\}} d M_{r}^{1} \\
& =\left(u_{1} \wedge u_{2}\right)(x)-C_{t}-\int_{0}^{t} f\left(\left(u_{1} \wedge u_{2}\right)\left(X_{r}\right)\right) d r \\
& -\int_{0}^{t} \mathbf{1}_{\left\{u_{1}\left(X_{r}\right)>u_{2}\left(X_{r}\right)\right\}} d A_{r}^{\mu_{2}}+\int_{0}^{t} \mathbf{1}_{\left\{u_{2}\left(X_{r}\right) \geq u_{2}\left(X_{r}\right)\right\}} d A_{r}^{\mu_{1}} \\
& +\int_{0}^{t} \mathbf{1}_{\left\{u_{1}\left(X_{r}\right)>u_{2}\left(X_{r}\right)\right\}} d A_{r}^{\nu_{2}}+\int_{0}^{t} \mathbf{1}_{\left\{u_{2}\left(X_{r}\right) \geq u_{2}\left(X_{r}\right)\right\}} d A_{r}^{\nu_{1}} \\
& +\int_{0}^{t} \mathbf{1}_{\left\{u_{1}\left(X_{r-)}>u_{2}\left(X_{r-}\right)\right\}\right.} d M_{r}^{2}+\int_{0}^{t} \mathbf{1}_{\left\{u_{2}\left(X_{r-}\right) \geq u_{2}\left(X_{r-}\right)\right\}} d M_{r}^{1},
\end{aligned}
$$

$t \in\left[0, \tau_{D}\right], P_{x}$-a.s. From the above formula, we get, in particular, that $C$ is a positive AF of $\mathbb{X}^{D}$. By [14, Theorem A.3.16] there exists a positive AF $C^{p}$ which is the dual predictable projection of $C$ under measure $P_{x}^{D}$ for q.e. $x \in D$. Since $\mathbb{X}^{D}$ is a Hunt process it is, by the very definition, quasi-left continuous, so it has only totally inaccessible jumps. Therefore, since $u_{1} \wedge u_{2}$ is quasi-continuous, process $\left(u_{1} \wedge u_{2}\right)(X)$ has only totally inaccessible jumps under measure $P_{x}^{D}$ for q.e. $x \in D$ (see [14, Theorem 4.2.2]). Moreover, by [6, Proposition 2 , Proposition 4] every local $\left(\mathcal{F}_{t}\right)_{t \geq 0}$-martingale has only totally inaccessible jumps. By the definition of dual predictable projection, $C^{p}-C$ is an $\left(\mathcal{F}_{t}\right)_{t \geq 0}$-martingale. Therefore, by Eq. 3.3, $C^{p}$ has only totally inaccessible jumps under measure $P_{x}^{D}$ for q.e. $x \in D$. However, $C^{p}$ is predictable. Consequently, $C^{p}$ is continuous. So, $C^{p}$ is a PCAF of $\mathbb{X}^{D}$. Hence $C^{p}=$ $A^{\beta}$ for some positive $\beta \in \mathbb{M}_{0}(D)$ (cf. Section 2.4). Define $\mu=\mathbf{1}_{u_{1}>u_{2}} \cdot \mu_{2}+\mathbf{1}_{u_{2} \geq u_{1}} \cdot \mu_{1}+\beta$ and $v=\mathbf{1}_{u_{1}>u_{2}} \cdot v_{2}+\mathbf{1}_{u_{2} \geq u_{1}} \cdot v_{1}$. By Lemma 2.1, for q.e $x \in D$,

$$
\left(u_{1} \wedge u_{2}\right)(x)=R^{D} f\left(u_{1} \wedge u_{2}\right)(x)+R^{D} \mu(x)-R^{D} v(x) .
$$

Let $\eta$ be a quasi-continuous function such that $u_{1} \wedge u_{2} \leq \eta \leq h m$-a.e. Observe that

$$
\begin{aligned}
\int_{D}\left(\eta-u_{1} \wedge u_{2}\right) d v & =\int_{u_{1}>u_{2}}\left(\eta-u_{2}\right) d v_{2}+\int_{u_{1} \leq u_{2}}\left(\eta-u_{1}\right) d v_{1} \\
& \leq \int_{u_{1}>u_{2}}\left(\eta \vee u_{2}-u_{2}\right) d v_{2}+\int_{u_{1} \leq u_{2}}\left(\eta \vee u_{1}-u_{1}\right) d v_{1} \\
& \leq \int_{D}\left(\eta \vee u_{2}-u_{2}\right) d v_{2}+\int_{D}\left(\eta \vee u_{1}-u_{1}\right) d v_{1}
\end{aligned}
$$


Clearly, $u_{2} \leq \eta \vee u_{2} \leq h m$-a.e., and $u_{1} \leq \eta \vee u_{1} \leq h m$-a.e. So, by condition (c) of Definition 3.1 applied to $\left(u_{1}, v_{1}\right)$ and $\left(u_{2}, v_{2}\right)$, we get

$$
\int_{D}\left(\eta \vee u_{2}-u_{2}\right) d \nu_{2}+\int_{D}\left(\eta \vee u_{1}-u_{1}\right) d \nu_{1}=0
$$

Thus, $\int_{D}\left(\eta-u_{1} \wedge u_{2}\right) d v=0$. This combined with Eq. 3.4 implies that $u_{1} \wedge u_{2}$ is an integral supersolution to Eq. 3.1.

Proposition 3.8 Assume that $f$ is nondecreasing. Let $\underline{u}$ (resp. $\bar{u}$ ) be a bounded integral subsolution (resp. supersolution) to Eq. 3.1 and $\underline{u} \leq \bar{u}$ q.e. Then there exists an integral solution u to Eq. 3.1 such that $\underline{u} \leq u \leq \bar{u}$ q.e.

Proof Let $u_{0}=\underline{u}$. We first show that for each $n \geq 1$ there exists an integral solution $u_{n}$ to the problem

$$
\left\{\begin{array}{l}
\max \left\{-\left(\Delta^{\alpha / 2}\right)_{\mid D} v-f\left(u_{n-1}\right), v-h\right\}=0 \\
v>0 \text { on } D
\end{array}\right.
$$

and

$$
u_{n} \leq u_{n+1} \text {, q.e., } \quad v_{n} \leq v_{n+1} \quad \underline{u} \leq u_{n} \leq \bar{u} \text {, q.e. } n \geq 1,
$$

where $v_{n}$ is the reaction measure for $u_{n}$. Indeed, the existence of $u_{1}$ follows from [19, Theorem 3.8]. By [19, Proposition 3.12], $\underline{u} \leq u_{1} \leq \bar{u}$ q.e. In particular, $R^{D}\left|f\left(u_{1}\right)\right|<\infty$ q.e. Hence, by [19, Theorem 3.8] again, there exists an integral solution $u_{2}$ to Eq .3.5, and by [19, Proposition 3.12] again, $\underline{u} \leq u_{2} \leq \bar{u}$ q.e. and $u_{1} \leq u_{2}$ q.e. Continuing in this fashion, we get the existence of $\left\{u_{n}\right\}$ having the desired properties. Moreover, by [19, Proposition 3.12], $v_{n} \leq v_{n+1}, n \geq 1$. Let $u=\sup _{n \geq 1} u_{n}$, and

$$
v(B):=\lim _{n} v_{n}(B), \quad B \in \mathcal{B}(D) .
$$

Observe that

$$
R^{D} v_{n} \leq R^{D} f\left(u_{n-1}\right) \leq R^{D}|f(\bar{u})|+R^{D}|f(\underline{u})|, \quad \text { q.e. }
$$

Therefore, by [21, Lemma 5.4],

$$
\left\|v_{n}\right\|_{T V} \leq\|f(\underline{u})\|_{L^{1}}+\|f(\bar{u})\|_{L^{1}}, \quad n \geq 1 .
$$

The right-hand side is finite since $\underline{u}, \bar{u}$ are bounded and $D$ is bounded. By the Vitali-HahnSaks theorem $v$ is a bounded Borel measure and

$$
\int_{D} f d v_{n} \rightarrow \int_{D} f d v, \quad f \in L^{1}(D ; v) .
$$

By Eq. 2.7, $R^{D} v<\infty$ q.e., and $R^{D}|f(\bar{u})|+R^{D}|f(\underline{u})|<\infty$ q.e. So, by Eq. 3.6 and the Lebesgue dominated convergence theorem,

$$
R^{D} v_{n} \rightarrow R^{D} v, \quad R^{D} f\left(u_{n-1}\right) \rightarrow R^{D} f(u), \quad \text { q.e. }
$$

Thus,

$$
u=R^{D} f(u)-R^{D} v, \quad \text { q.e. }
$$

By Remark 3.2(iv), $u$ is quasi-continuous. Clearly, $u \geq h, m$-a.e. Let $\eta$ be an arbitrary quasi-continuous function such that $u \leq \eta \leq h m$-a.e. Then, by the minimality condition (c) of Definition 3.1 applied to $\left(u_{n}, v_{n}\right)$, and by Eq. 3.6,

$$
0=\int_{D}\left(\eta-u_{n}\right) d v_{n} \geq \int_{D}(\eta-u) d v_{n} \rightarrow \int_{D}(\eta-u) d v \geq 0 .
$$

Thus, $u$ is an integral solution to Eq. 3.1. 


\section{Uniqueness Result}

As in Sections 2 and 3, we assume that $D$ is a bounded Lipschitz domain in $\mathbb{R}^{d}(d \geq 2)$ and $D_{0} \subset D$ is a bounded Dirichlet regular domain. We assume that $a>\lambda_{1}^{D}$, where $\lambda_{1}^{D}$ is the first eigenvalue for the operator $-\left(\Delta^{\alpha / 2}\right)_{\mid D}$. By $\varphi_{1}^{D}$ we denote the ground state for $-\left(\Delta^{\alpha / 2}\right)_{\mid D}$, i.e. a unique strictly positive function $\varphi_{1}^{D} \in H_{0}^{\alpha / 2}(D)$ such that $\left\|\varphi_{1}^{D}\right\|_{L^{2}}=1$ and

$$
\mathcal{E}_{D}\left(\varphi_{1}^{D}, \eta\right)=\lambda_{1}^{D}\left(\varphi_{1}^{D}, \eta\right), \quad \eta \in D\left(\mathcal{E}_{D}\right) .
$$

It is well known that $\lambda_{1}^{D}>0$, and by the regularity of $D, \varphi_{1}^{D} \in C_{0}(D)$.

To prove a uniqueness result for Eq. 1.1, we shall need some regularity results for integral solutions to Eq. 1.1, and the result which compare the Green function $G_{D}$ for $\left(\Delta^{\alpha / 2}\right)_{\mid D}$ with the Green function $G_{D}^{v}$ for $\left(\Delta^{\alpha / 2}\right)_{\mid D}-v$.

It is well known (see e.g. [15]) that the semigroup $\left(P_{t}^{D}\right)_{t>0}$ is intrinsically ultracontractive, which implies that for every $t>0$ there exist constants $c_{1}(t), c_{2}(t)>0$ such that

$$
c_{1}(t) \varphi_{1}^{D}(x) \varphi_{1}^{D}(y) \leq p_{D}(t, x, y) \leq c_{2}(t) \varphi_{1}^{D}(x) \varphi_{1}^{D}(y), \quad x, y \in D .
$$

Proposition 4.1 Let $u$ be an integral solution to Eq. 1.1 and $v$ be the reaction measure for $u$. Then $u \cdot v=v$.

Proof Set $w=R^{D} u, w_{n}=R^{D}(u \wedge n)$, and

$$
h(x)=1+a \rho(x), \quad \rho(x)=\mathbb{E}_{x} \int_{0}^{\tau_{D_{0}}} u\left(X_{r}\right) d r, \quad x \in D .
$$

Clearly, $w_{n} \in H_{0}^{\alpha / 2}(D)$ since $u \wedge n \in L^{2}(D ; m)$. By Dynkin's formula (see [14, (4.4.2)]),

$$
\rho(x)+\mathbb{E}_{x}\left[w\left(X_{\tau_{D_{0}}}\right)\right]=R^{D} u(x), \quad x \in D .
$$

By $\left[14\right.$, Lemma 4.3.1], $\mathbb{E}$. $\left[w_{n}\left(X_{\tau_{D_{0}}}\right)\right]$ is an excessive function for $n \geq 1$. So, by [3, Proposition II.2.2], $\mathbb{E} .\left[w\left(X_{\tau_{D_{0}}}\right)\right]$ is an excessive function. By the definition of an integral solution, $u \in L^{1}(D ; m)$. Therefore, by Eq. $2.7, R^{D} u$ is finite q.e. So, by Eq. $4.3, \mathbb{E}$. $\left[w\left(X_{\tau_{D_{0}}}\right)\right]$ is finite q.e. Consequently, $\rho$ as a difference of excessive functions finite q.e. is quasicontinuous (see comments in Remark 3.2(iv)). Thus, $h$ is quasi-continuous. By regularity of $D_{0}, \rho(x)=0, x \in D \backslash D_{0}$. Moreover,

$$
\rho(x)=\int_{D} G_{D_{0}}(x, y) u(y) m(d y)>0, \quad x \in D_{0} .
$$

By Dynkin's formula again

$$
u(x)=\mathbb{E}_{x} u\left(X_{\tau_{D_{0}}}\right)+a \mathbb{E}_{x} \int_{0}^{\tau_{D_{0}}} u\left(X_{r}\right) d r-\mathbb{E}_{x} \int_{0}^{\tau_{D_{0}}} d A_{r}^{v} \leq 1+a \rho(x)=h(x), \quad \text { q.e. }
$$

From this and the fact that $\rho=0$ on $D \backslash D_{0}$, we get that the $n$-th power of $h$ satisfies

$$
u \leq h^{n} \leq \mathbb{I}_{D \backslash \bar{D}_{0}}, \quad n \geq 1, \quad \text { q.e. }
$$

Hence, by the definition of an integral solution to the obstacle problem,

$$
\int_{D}\left(h^{n}-u\right) d v=0, \quad n \geq 1 .
$$


By Eq. 4.4, for every $x \in D_{0}, h^{n}(x) \nearrow \infty$ as $n \rightarrow \infty$. It follows from Eq. 4.5 that supp $[v] \subset D \backslash D_{0}$. By this and Eq. 4.5 again, for every positive $\eta \in C_{c}(D)$ we have

$$
0=\int_{D} \eta(h-u) d v=\int_{D \backslash D_{0}} \eta(h-u) d v=\int_{D \backslash D_{0}} \eta(1-u) d v=\int_{D} \eta(1-u) d v,
$$

which implies the desired result.

Proposition 4.2 If $u$ is an integral solution to Eq. 1.1, then

(i) $u(x) \leq c\|u\|_{L_{1}(D ; m)} \varphi_{1}^{D}(x)$ for q.e. $x \in D$.

(ii) $u \in C_{0}(D)$.

(iii) The reaction measure $v$ for $u$ is bounded and $\|v\|_{T V} \leq a\|u\|_{L^{1}(D ; m)}$.

Proof By the definition of an integral solution to Eq. 1.1 and Proposition 4.1,

$$
u(x)=a \mathbb{E}_{x} \int_{0}^{\tau_{D}} u\left(X_{r}\right) d r-\mathbb{E}_{x} \int_{0}^{\tau_{D}} u\left(X_{r}\right) d A_{r}^{\nu}
$$

for q.e $x \in D$. Using Lemma 2.1 and the integration by part formula applied to the product $e^{a t-A_{t}^{v}} u\left(X_{t}\right)$ yields

$$
u(x)=e^{a t} \mathbb{E}_{x} e^{-A_{t}^{v}} u\left(X_{t}\right), \quad t \geq 0,
$$

for q.e. $x \in D$. Therefore, by the ultracontractivity of $\left(P_{t}^{D}\right)_{t \geq 0}$, for every $t>0$,

$$
u(x) \leq e^{a t} \int_{D} p_{D}(t, x, y) u(y) d y \leq c_{t} e^{a t} \varphi_{1}^{D}(x)\|u\|_{L^{1}(D ; m)}
$$

for q.e. $x \in D$, which proves (i). To prove (ii), consider the function $h$ defined by Eq. 4.2. By regularity of the set $D_{0}$ and [5], $\left(P_{t}^{D_{0}}\right)_{t \geq 0}$ is doubly Feller (cf. Section 2.4). Therefore, by (i) $\rho \in C\left(D_{0}\right)$. By regularity of $D_{0}$, in fact $\rho \in C_{0}\left(D_{0}\right)$. Thus, $h \in C(\bar{D})$. By the proof of Proposition 4.1,

$$
u \leq h \leq \mathbb{I}_{D \backslash \bar{D}_{0}}, \quad \text { q.e. }
$$

So, by Remark 3.2(i), $u$ is an integral solution to Eq. 1.1 with $\mathbb{I}_{D \backslash \bar{D}_{0}}$ replaced by $h$. Therefore, $u \in C_{0}(D)$ by [28, Theorem 1], which proves (ii). By the definition of an integral solution to Eq. 1.1,

$$
\mathbb{E}_{x} \int_{0}^{\tau_{D}} d A_{r}^{v} \leq a \mathbb{E}_{x} \int_{0}^{\tau_{D}} u\left(X_{r}\right) d r
$$

for q.e. $x \in D$. From Eq. 4.6 and [21, Lemma 5.4] we get (iii).

Proposition 4.3 Let $u$ be an integral solution to Eq. 1.1 and $v$ be its reaction measure. Then

(i) $\operatorname{supp}[v]$ is a compact subset of $D$.

(ii) $\sup _{x \in D} R^{D} v(x)<\infty$.

Proof By Proposition 4.1, supp[v] $\subset\{u=1\}$, which when combined with Proposition 4.2(ii) implies (i). Assertion (ii) follows easily from Eq. 4.6, Proposition 4.2 and Eq. 2.9.

Proposition 4.4 Let $u$ be an integral solution to Eq. 1.1 and let $v$ be its reaction measure. Then $u \in H_{0}^{\alpha / 2}(D), v \in S_{0}(D)$ and for every $\eta \in H_{0}^{\alpha / 2}(D)$,

$$
\mathcal{E}_{D}(u, \eta)=a(u, \eta)-\int_{D} \tilde{\eta} d \nu .
$$


Proof By Proposition 4.2, $u \in L^{1}(D ; m)$ and $v \in \mathcal{M}_{0, b}(D)$. Therefore, by [22, Theorem 3.5], $u$ is a renormalized solution to (1.1) in the sense defined in [22]. By the definition of a renormalized solution, $u \wedge k \in H_{0}^{\alpha / 2}(D), k \geq 1$, which when combined with Proposition 4.2(i) implies that $u \in H_{0}^{\alpha / 2}(D)$. Moreover, by the definition of a renormalized solution, there exists a sequence $v_{k} \subset \mathcal{M}_{0, b}(D)$ such that $\left\|v_{k}\right\|_{T V} \rightarrow 0$ and for every bounded $\eta \in H_{0}^{\alpha / 2}(D)$

$$
\mathcal{E}_{D}(u \wedge k, \eta)+\int_{D} \tilde{\eta} d v=a(u, \eta)+\int_{D} \tilde{\eta} d v_{k}
$$

Letting $k \rightarrow \infty$ yields (4.7) for every bounded $\eta \in H_{0}^{\alpha / 2}(D)$. Applying now a simple approximation argument, we get that $v \in S_{0}(D)$, and Eq. 4.7 holds for any $\eta \in H_{0}^{\alpha / 2}(D)$.

Let $w$ be a strictly positive excessive function with respect to $\left(P_{t}^{D}\right)_{t \geq 0}$. We say that $G_{D}$ has $w$-triangle property iff there exists $C>0$ such that

$$
G_{D}(x, z) G_{D}(z, y) \leq C G_{D}(x, y) \max \left(\frac{w(z)}{w(x)} G_{D}(x, z), \frac{w(z)}{w(y)} G_{D}(z, y)\right), \quad x, y, z \in D .
$$

The above notion was introduced in [16]. Observe that if we set

$$
\rho(x, y):=\frac{w(x) w(y)}{G_{D}(x, y)}, \quad x, y \in D
$$

then Eq. 4.8 is equivalent to

$$
\rho(x, y) \leq C \max (\rho(x, z), \rho(z, y)), \quad x, y, z \in D .
$$

Clearly, $\rho(x, y)=0$ if and only if $x=y$, and $\rho(x, y)=\rho(y, x)$. Therefore, $w$-triangle property is equivalent to the statement that $\rho$ is a quasi-metric on $D$.

Recall here that at the beginning of Section 2, we introduced $\lambda>0$ and $r_{0}>0$. We shall show that $G_{D}$ has $w$-triangle property for Lipschitz domains, where $w=\phi$ and

$$
\phi(x)=\min \left(G_{D}\left(x, x_{0}\right), c_{d, \alpha}\left(r_{0} / 4\right)^{\alpha-d}\right), \quad x \in D
$$

for a fixed $x_{0} \in D$. Here $c_{d, \alpha}=\frac{\Gamma((d-\alpha) / 2)}{2^{\alpha} \pi^{d / 2}|\Gamma(\alpha / 2)|}$. Set $\kappa=1 / 2 \sqrt{1+\lambda^{2}}$, and fix $x_{1} \in D$ such that $\left|x_{0}-x_{1}\right|=r_{0} / 4$. We let $\delta_{D}(x):=\operatorname{dist}(x, \partial D), x \in D$.

Proposition 4.5 Green function $G_{D}$ has $\phi$-triangle property.

Proof Let $x, y, z, z_{1}, z_{2} \in D$. By [17, Theorem 1],

$$
c_{1} \frac{\phi(x) \phi\left(z_{i}\right)}{\phi^{2}\left(A_{i}\right)} \leq G_{D}\left(x, z_{i}\right) \leq c_{2} \frac{\phi(x) \phi\left(z_{i}\right)}{\phi^{2}\left(A_{i}\right)},
$$

for $A_{i} \in \mathfrak{B}\left(x, z_{i}\right), i=1,2$, where $c_{1}, c_{2}$ depend only on $D, d, \lambda, \alpha$. Here, for $r_{i}:=\delta_{D}(x) \vee$ $\delta_{D}\left(z_{i}\right) \vee\left|x-z_{i}\right| \leq r_{0} / 32$,

$$
\mathfrak{B}\left(x, z_{i}\right)=\left\{A \in D: B\left(A, \kappa r_{i}\right) \subset D \cap B\left(x, 3 r_{i}\right) \cap B\left(z_{i}, 3 r_{i}\right)\right\},
$$

and $\mathfrak{B}\left(x, z_{i}\right)=\left\{x_{1}\right\}$ for $r_{i}>r_{0} / 32$. Suppose that $|x-z| \geq|y-z|$. Set $z_{1}:=y, z_{2}:=z$. By Eq. 4.10,

$$
\frac{G_{D}(x, z)}{G_{D}(x, y)}=\frac{G_{D}\left(x, z_{2}\right)}{G_{D}\left(x, z_{1}\right)} \leq \frac{c_{2}}{c_{1}} \frac{\phi\left(z_{2}\right)}{\phi\left(z_{1}\right)}\left(\frac{\phi\left(A_{1}\right)}{\phi\left(A_{2}\right)}\right)^{2}=\frac{c_{2}}{c_{1}} \frac{\phi(z)}{\phi(y)}\left(\frac{\phi\left(A_{1}\right)}{\phi\left(A_{2}\right)}\right)^{2} .
$$


Observe that $\left|x-z_{1}\right| \leq 2\left|x-z_{2}\right|$. Therefore, by [17, Lemma 13], there exists $C_{1}>0$ such that $\frac{\phi\left(A_{1}\right)}{\phi\left(A_{2}\right)} \leq C_{1}$, where $C_{1}$ depends only on $\alpha, d$ and $D$. Consequently, there exists $C>0$ such that

Equivalently,

$$
\frac{G_{D}(x, z)}{G_{D}(x, y)} \leq C \frac{\phi(z)}{\phi(y)}, \quad x, y, z \in D ;|x-z| \geq|y-z| .
$$

$$
\rho(x, y) \leq C \rho(x, z), \quad x, y, z \in D ;|x-z| \geq|y-z| .
$$

Hence we get easily Eq. 4.9.

Let $G$ denote the Green function for $\mathbb{R}^{d}$ (and the operator $\Delta^{\alpha / 2}$ ). It is well known that there is $c>0$ such that

$$
G(x, y)=\frac{c}{|x-y|^{d-\alpha}}, \quad x, y \in \mathbb{R}^{d} .
$$

Lemma 4.6 Assume that $\mu \in \mathcal{M}_{0, b}(D)$ is positive, $K:=\operatorname{supp}[\mu]$ is a compact subset of $D$ and $\sup _{x \in D} R^{D} \mu(x)<\infty$. Then $G_{D}^{\mu} \sim G_{D}$, i.e. there exist $c_{1}, c_{2}>0$ such that $c_{1} G_{D}^{\mu} \leq G_{D} \leq c_{2} G_{D}^{\mu}$ on $D \times D$.

Proof Let $\bar{\mu}$ be an extension of $\mu$ to $\mathbb{R}^{d}$ defined as $\bar{\mu}(B)=\mu(D \cap B)$ for any Borel set $B \subset \mathbb{R}^{d}$. Let $V$ be an open set such that $K \subset V \subset \bar{V} \subset D$, and let $r=\operatorname{dist}(K, \partial V)$. By [4, Theorem 1.2], there is $c>0$ such that

$$
c G(x, y) \leq G_{D}(x, y), \quad x, y \in V .
$$

From this and the assumptions of the lemma it follows that

$$
\begin{aligned}
\sup _{x \in D} R \bar{\mu}(x) & \leq \max \left\{\sup _{x \in V} R \bar{\mu}(x), \sup _{x \in D \backslash V} R \bar{\mu}(x)\right\} \\
& \leq \max \left\{c \sup _{x \in V} R^{D} \mu(x), \sup _{x \in D \backslash V} \int_{K} G(x, y) \mu(d y)\right\} \\
& \leq c \max \left\{\sup _{x \in D} R^{D} \mu(x), r^{\alpha-d}\|\mu\|_{T V}\right\} .
\end{aligned}
$$

Next, for all $x, y \in D$,

$$
\begin{aligned}
\int_{D} \frac{G(x, z) G(z, y)}{G(x, y)} \mu(d z) & =\int_{D} \frac{|x-y|^{d-\alpha}}{|x-z|^{d-\alpha}|z-y|^{d-\alpha}} \mu(d z) \\
& \leq 2^{d-\alpha} \int_{D} \frac{\max \left\{|x-z|^{d-\alpha},|z-y|^{d-\alpha}\right\}}{|x-z|^{d-\alpha}|z-y|^{d-\alpha}} \mu(d z) \\
& \leq 2^{d-\alpha} R \bar{\mu}(x)+2^{d-\alpha} R \bar{\mu}(y) .
\end{aligned}
$$

By the 3G Theorem (see [18]),

$$
\int_{D} \frac{G_{D}(x, z) G_{D}(z, y)}{G_{D}(x, y)} \mu(d z) \leq c \int_{D} \frac{G(x, z) G(z, y)}{G(x, y)} \mu(d z), \quad x, y \in D .
$$

This when combined with Eqs. 4.11 and 4.12 shows that there exists $C>0$ such that

$$
\int_{D} G_{D}(x, z) G_{D}(z, y) \mu(d z) \leq C G_{D}(x, y), \quad x, y \in D .
$$

From this we conclude that for every positive Borel measure $v$ on $D$,

$$
\int_{D} G_{D}(x, z)\left(\int_{D} G_{D}(z, y) v(d y)\right) \mu(d z) \leq C \int_{D} G_{D}(x, y) v(d y), \quad x, y \in D .
$$


Equivalently,

$$
\int_{D} G_{D}(x, z) R^{D} v(z) \mu(d z) \leq C R^{D} v(x), \quad x, y \in D .
$$

It is well known (see [13, Theorem 17, page 230]) that each $\left(P_{t}^{D}\right)_{t \geq 0}$-excessive function is an increasing limit of functions of the form $R^{D} v$ for some positive Borel measure $v$. Therefore from (4.14) it follows that for every excessive function $e$,

$$
\int_{D} G_{D}(x, z) e(z) \mu(d z) \leq C e(x), \quad x, y \in D .
$$

Taking $e=\phi$ (it is excessive as a minimum of excessive functions), we get

$$
\sup _{x \in D} \int_{D} \frac{\phi(z)}{\phi(x)} G_{D}(x, z) \mu(d z)<\infty .
$$

From this, Proposition 4.5, and [16, Theorem 9.1] we get the desired result.

Now, we shall proceed to the proof of the main result, i.e. the uniqueness for integral/weak solutions to Eq. 1.1 (see Definition 3.1 and Proposition 3.8). Recall that $d \geq 2$, $\alpha \in(0,2), D_{0}, D$ are bounded domains in $\mathbb{R}^{d}$ which are assumed to be Dirichlet regular (see the beginning of Section 2.4) and Lipschitz (see the introductory part of Section 2), respectively, and $D_{0} \subset D$. Recall also that $\lambda_{1}^{D_{0}}, \lambda_{1}^{D}$ stand for the principal eigenvalues for $\left(\Delta^{\alpha / 2}\right)_{\mid D_{0}},\left(\Delta^{\alpha / 2}\right)_{\mid D}$, respectively (see the beginning of Section 4 ).

Theorem 4.7 Assume that $a \in\left(\lambda_{1}^{D}, \lambda_{1}^{D_{0}}\right)$. Then there exists at most one integral solution to Eq. 1.1 .

Proof Let $u_{1}, u_{2}$ be two integral solutions to Eq. 1.1. We divide the proof into two steps.

Step 1. We shall show that without loss of generality we may assume that $u_{1} \leq u_{2}$. Assume that whenever we know that $w, v$ are integral solutions to Eq. 1.1 such that $w \leq v$, then $w=v$. By Proposition 3.8, $u:=u_{1} \wedge u_{2}$ is a an integral supersolution to Eq. 1.1. It is clear that for a sufficiently small $c>0, c \varphi_{1}^{D} \leq \mathbb{I}_{D \backslash \bar{D}_{0}}$. Therefore, since

$$
-\left(\Delta^{\alpha / 2}\right)_{\mid D}\left(c \varphi_{1}^{D}\right)=\lambda_{1}^{D} c \varphi_{1}^{D}=a c \varphi_{1}^{D}-c\left(a-\lambda_{1}^{D}\right) \varphi_{1}^{D}
$$

and $a>\lambda_{1}^{D}$, we see that $c \varphi_{1}^{D}$ is a an integral subsolution to Eq. 1.1 (cf. Eq. 2.8). By the definition of an integral supersolution to Eq. 1.1, there exist positive measures $\mu, v \in$ $\mathbb{M}_{0}(D)$ such that

$$
u=a R^{D} u+R^{D} \mu-R^{D} v
$$

and $v$ is the reaction measure for $u$. By Proposition 4.1 , the above equation may be equivalently rewritten as

$$
u=a R^{D} u+R^{D} \mu-R^{D}(u \cdot v) .
$$

By Lemma 2.1 there exists a MAF $M$ such that for any $x \in D$,

$u\left(X_{t}\right)=\int_{t}^{\tau_{D}} a u\left(X_{r}\right) d r+\int_{t}^{\tau_{D}} d A_{r}^{\mu}-\int_{t}^{\tau_{D}} u\left(X_{r}\right) d A_{r}^{v}-\int_{t}^{\tau_{D}} d M_{r}, \quad t \in\left[0, \tau_{D}\right], \quad P_{x}$-a.s.

By the integration by parts formula applied to the product $e^{-A_{t}} u\left(X_{t}\right)$, we get

$$
e^{-A_{t}^{v}} u\left(X_{t}\right)=\int_{t}^{\tau_{D}} a e^{-A_{r}^{v}} u\left(X_{r}\right) d r+\int_{t}^{\tau_{D}} e^{-A_{r}^{v}} d A_{r}^{\mu}-\int_{t}^{\tau_{D}} e^{-A_{r}^{v}} d M_{r}, \quad t \in\left[0, \tau_{D}\right],
$$


$P_{x}$-a.s. Taking expectation $\mathbb{E}_{x}$ of both sides of the above equation with $t=0$ yields

$$
u(x)=\mathbb{E}_{x} \int_{0}^{\tau_{D}} a e^{-A_{r}^{v}} u\left(X_{r}\right) d r+\mathbb{E}_{x} \int_{0}^{\tau_{D}} e^{-A_{r}^{v}} d A_{r}^{\mu}, \quad x \in D .
$$

Therefore, by Eq. 2.15,

$$
u(x)=a \int_{D} G_{D}^{v}(x, y) u(y) d y+\int_{D} G_{D}^{v}(x, y) \mu(d y), \quad x \in D .
$$

By Proposition 4.2, Proposition 4.3 and Lemma 4.6, $G_{D}^{v} \sim G_{D}$. This when combined with the above equation and (4.1) gives

$$
u(x) \geq a c \int_{D} G_{D}(x, y) u(y) d y \geq \operatorname{acc}_{1} \varphi_{1}^{D}(x) \int_{D} \varphi_{1}^{D}(y) u(y) d y, \quad x \in D,
$$

so $u \geq c \varphi_{1}^{D}$ for some $c>0$. Hence, for a sufficiently small $c>0, c \varphi_{1}^{D}$ is a an integral subsolution to Eq. 1.1 such that $c \varphi_{1}^{D} \leq u$. By Proposition 3.8, there exists an integral solution $v$ to Eq. 1.1 such that $c \varphi_{1}^{D} \leq v \leq u$. Hence $v \leq u_{1}$ and $v \leq u_{2}$. By the assumption of Step $1, u_{1}=v=u_{2}$.

Step 2. Assume that $u_{1} \leq u_{2}$. Let $v_{1}, v_{2}$ be the reaction measures for $u_{1}$ and $u_{2}$, respectively. Then, by Proposition 4.4,

$$
\mathcal{E}_{D}\left(u_{1}, u_{2}\right)+\int_{D} u_{2} d v_{1}=a\left(u_{1}, u_{2}\right), \quad \mathcal{E}_{D}\left(u_{2}, u_{1}\right)+\int_{D} u_{1} d v_{2}=a\left(u_{2}, u_{1}\right)
$$

Hence

$$
\int_{D} u_{2} d v_{1}-\int_{D} u_{1} d v_{2}=0
$$

From this and Proposition 4.1 we conclude that

$$
\int_{D} u_{1} u_{2}\left(d \nu_{1}-d \nu_{2}\right)=0
$$

We can regard $u_{i}$ as a solution to the following obstacle problem

$$
\max \left\{-\left(\Delta^{\alpha / 2}\right)_{\mid D} w_{i}-g_{i}, w_{i}-\mathbb{I}_{D \backslash \bar{D}_{0}}\right\}=0,
$$

where $g_{i}=a u_{i}, i=1$, 2. Since $u_{1} \leq u_{2}$, we have $g_{1} \leq g_{2}$. Applying [19, Proposition 3.12] yields $d v_{1} \leq d v_{2}$. This when combined with (4.17) and the fact that $u_{1}, u_{2}$ are strictly positive implies that $v_{1}=v_{2}$. Therefore, we have

$$
\left(u_{2}-u_{1}\right)=a R^{D}\left(u_{2}-u_{1}\right) .
$$

Thus,

$$
\left(u_{2}-u_{1}\right)(x)=a \int_{D} G_{D}(x, y)\left(u_{2}-u_{1}\right)(y) d y, \quad x \in D .
$$

We have assumed that $u_{2}-u_{1} \geq 0$. Striving for a contradiction, suppose that $\left(u_{2}-u_{1}\right)(x)>$ 0 for some $x \in D$. Then continuity of $u_{1}, u_{2}$ and (4.18) would imply that $u_{2}-u_{1}$ is strictly positive on $D$, in contradiction with the fact that $a>\lambda_{1}^{D}$.

Remark 4.8 All the results of the paper hold for $\alpha=2$. In case $\alpha=2$ the proofs run in the same way as in case $\alpha \in(0,2)$, the only difference being in the proof of Proposition 4.5 and Lemma 4.6. In case $d \geq 3$, Proposition 4.5 follows from [26, Theorem 3.1], and in case $d=2$ it follows from [16, Corollary 9.6] - we need however $D$ to be finitely connected. As 
to the proof of Lemma 4.6, in case $d=2$, to get (4.13), we use [7, Theorem 6.24], and in case $d \geq 3$, we use [7, Theorem 6.5]. Instead of [4, Theorem 1.2], we use [7, Lemma 6.7].

Ultracontractivity of the semigroup generated by $\Delta_{D}$ follows from [12, Theorem 9.3]. That Lipschitz bounded domains are Dirichlet regular is well known (see, e.g., [2, page 350]).

Acknowledgements This work was supported by Polish National Science Centre(Grant No. 2017/25/B/ST1/00878). Data sharing not applicable to this article as no datasets were generated or analysed during the current study.

Open Access This article is licensed under a Creative Commons Attribution 4.0 International License, which permits use, sharing, adaptation, distribution and reproduction in any medium or format, as long as you give appropriate credit to the original author(s) and the source, provide a link to the Creative Commons licence, and indicate if changes were made. The images or other third party material in this article are included in the article's Creative Commons licence, unless indicated otherwise in a credit line to the material. If material is not included in the article's Creative Commons licence and your intended use is not permitted by statutory regulation or exceeds the permitted use, you will need to obtain permission directly from the copyright holder. To view a copy of this licence, visit http://creativecommons.org/licenses/by/4.0/.

\section{References}

1. Billingsley, P.: Convergence of probability measures. Second edition. Wiley Series in Probability and Statistics: Probability and Statistics. A Wiley-Interscience Publication. Wiley, New York, (1999)

2. Bliedtner, J., Hansen, W.: Potential Theory. Springer, Berlin (1986)

3. Blumenthal, M.R., Getoor, R.K.: Markov Processes and Potential Theory. Dover Publications, New York (2007)

4. Chen, Z.Q., Song, R.: Estimates on Green functions and Poisson kernels for symmetric stable processes. Math. Ann. 312, 465-501 (1998)

5. Chung, K.L.: Doubly-Feller Process with Multiplicative Functional. Seminar on Stochastic Processes, 1985 (Gainesville, Fla., 1985), 63-78, Progr. Probab Statist., 12, Birkhäuser, Boston, MA (1986)

6. Chung, K.L., Walsh, J.B.: Meyer's theorem on predictability. Z. Wahrscheinlichkeitstheorie und Verw. Gebiete 29, 253-256 (1974)

7. Chung, K.L., Zhao, Z.X.: From Brownian Motion to Schrödinger's Equation. Springer, Berlin (1995)

8. Dancer, E., Du, Y.: On a free boundary problem arising from population biology. Indiana Univ. Math. J. 52, 51-67 (2003)

9. Dancer, E., Du, Y.: A uniqueness theorem for a free boundary problem. Proc. Amer. Math. Soc. 134, 3223-3230 (2006)

10. Dancer, E., Du, Y., Ma, L.: Asymptotic behavior of positive integral solutions of some elliptic problems. Pacific J. Math. 210, 215-228 (2003)

11. Dancer, E.N., Hilhorst, D., Mimura, M., Peletier, L.A.: Spatial segregation limit of a competitiondiffusion system. Eur. J. Appl. Math. 10, 97-115 (1999)

12. Davies, E.B., Simon, B.: Ultracontractivity and the heat kernel for schrödinger operators and Dirichlet Laplacians. J. Funct. Anal. 59, 335-395 (1984)

13. Dellacherie, C., Meyer, P.A.: Probabilities and Potential C. North-Holland, Amsterdam (1988)

14. Fukushima, M., Oshima, Y., Takeda, M.: Dirichlet Forms and Symmetric Markov Processes. Second Revised and Extended Edition. Walter de Gruyter, Berlin (2011)

15. Grzywny, T.: Intrinsic ultracontractivity for lévy processes. Probab. Math. Statist. 28, 91-106 (2008)

16. Hansen, W.: Global comparison of perturbed Green functions. Math. Ann. 334, 643-678 (2006)

17. Jakubowski, T.: The estimates for the Green function in Lipschitz domains for the symmetric stable processes. Probab. Math. Statist. 22, 419-441 (2002)

18. Kim, P., Lee, Y.R.: Generalized 3G theorem and application to relativistic stable process on non-smooth open sets. J. Funct. Anal. 246, 113-143 (2007)

19. Klimsiak, T.: Quasi-regular Dirichlet forms and the obstacle problem for elliptic equations with measure data. Studia Math. 258, 121-156 (2021)

20. Klimsiak, T.: Asymptotics for logistic-type equations with Dirichlet fractional Laplace operator arXiv:1905.01667v2 (2021) 
21. Klimisak, T., Rozkosz, A.: Dirichlet forms and semilinear elliptic equations with measure data. J. Funct. Anal. 265, 890-925 (2013)

22. Klimsiak, T., Rozkosz, A.: Renormalized integral solutions of semilinear equations involving measure data and operator corresponding to Dirichlet form. NoDEA Nonlinear Differential Equations Appl. 22, 1911-1934 (2015)

23. Lions, J.-L.: Quelques méthodes de réintegral solutions des problèmes aux limites non linéaires. Dunod Gauthier Villars, Paris (1969)

24. McLean, W.: Strongly elliptic systems and boundary integral equations. Cambridge University Press, Cambridge (2000)

25. Protter, P.: Stochastic integration and differential equations second edition. Springer, Berlin (2004)

26. Riahi, L.: The 3G-inequality for general schrödinger operators on Lipschitz domains. Manuscripta Math. 116, 211-227 (2005)

27. Rodrigues, J.F., Tavares, H.: Increasing powers in a degenerate parabolic logistic equation. Chin. Ann. Math. Ser. B 34, 277-294 (2013)

28. Stettner, L.: Zero-sum Markov games with stopping and impulsive strategies. Appl. Math. Optim. 9, $1-24$ (1982)

Publisher's Note Springer Nature remains neutral with regard to jurisdictional claims in published maps and institutional affiliations. 